\title{
Towards energetically viable asymmetric deprotonations: selectivity at more elevated temperatures with $C_{2}$-symmetric magnesium bisamides $\dagger$
}

\author{
Linsey S. Bennie, William J. Kerr, * Michael Middleditch and Allan J. B. Watson
}

Received 12th November 2010, Accepted 22nd December 2010

DOI: $10.1039 /$ c0cc04939e

A novel chiral magnesium bisamide has enabled the development of effective asymmetric deprotonation protocols at substantially more elevated temperatures. This new, structurally simple, $C_{2}$-symmetric magnesium complex displays excellent levels of asymmetric efficiency and energy reduction in the synthesis of enantioenriched enol silanes.

The ability to perform intricate chemical manipulations is essential for the continued production of the myriad organic compounds that our society consumes. By their very nature, many of these transformations must be carried out at energy intensive temperatures. However, the energy expenditure of chemical processes is becoming a continually more important consideration, ${ }^{1}$ and with escalating environmental, financial, and political pressures, the demand for energetic efficiency within the chemical industry is only likely to increase. The financial impact of energy input is perhaps felt most dramatically when performing reactions on larger scale. ${ }^{2}$ Figures used within Chemical Development at GlaxoSmithKline suggest that performing reactions at temperatures of lower than $-40{ }^{\circ} \mathrm{C}$ incurs an additional energetic cost of at least $£ 250000$ per annum per batch tonne process. ${ }^{3}$ Moreover, this is supp lementary to the initial and appreciable expense of installing the necessary low temperature process scale equipment. Consequently, the task of alleviating the energetic burden of the chemical industry, through the continued development of more effective synthetic protocols that generate a smaller energy footprint than preceding technologies, is of global relevance. In this regard, contributions by several research groups have already illustrated the potential for considered experimental design in this area of enhanced sustainability. ${ }^{2,4,5}$ For example, the metallation processes pioneered by Knochel enable a more convenient and widely applicable preparation of nucleophilic organometallic species, ${ }^{4}$ while several groups have also focused on the development of catalyst systems that facilitate effective cross-coupling processes at more moderate temperatures. ${ }^{5}$ As part of a wider programme targeting the

Department of Pure and Applied Chemistry, WestCHEM,

295 Cathedral Street, Glasgow, G1 1XL, Scotland, UK.

E-mail:w.kerr@strath.ac.uk; Fax: +44(0)141548 4822;

Tel: +44 (0)1415482959

$\dagger$ Electronic supplementary information (ESI) available: Experimental procedures and analytical data for products. See DOI: 10.1039/ c0cc04939e production of effective methods for use in organic synthesis, a specific objective has been the design of more efficient and, indeed, energetically viable asymmetric deprotonation processes.

Asymmetric deprotonation reactions ${ }^{6}$ are useful methods for the preparation of enantioenriched chiral enolates and enol silanes, which are valuable precursors for a host of powerful diastereoselective transformations. ${ }^{7}$ Chiral lithium amides have been developed extensively in this area and several systems provide very good levels of enantioinduction at low reaction temperatures. ${ }^{6,8}$ In this regard, it should be noted that, while enantioselective deprotonation processes are typically conducted at $-78{ }^{\circ} \mathrm{C}$, several groups have demonstrated the ability of chiral lithium amides to afford high levels of selectivity at more elevated temperatures in the area of epoxide rearrangement. ${ }^{9}$ In addition to all of this, work within our laboratory has revealed that chiral magnesium amides are also particularly effective within the domain of asymmetric enolisation and, more significantly, were found to exhibit greater asymmetric efficiency than the equivalent lithium amides. ${ }^{10}$

In relation to the developing applications noted above, magnesium amides are known to possess greater thermal stability $^{11}$ and exhibit less complex solution aggregation than analogous lithium amides in THF (Fig. 1). ${ }^{12}$ As such, we recently began examining the potential for exploitation of these unique attributes to enable effective asymmetric induction at substantially more elevated temperatures. Herein, we

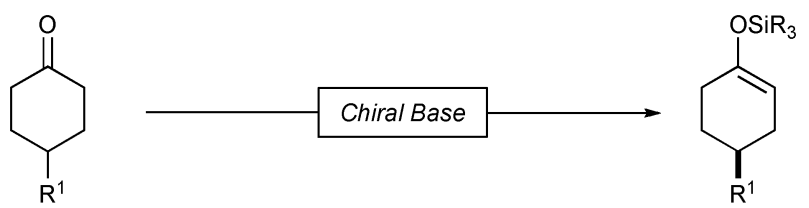

Lithium Bases: Oligomeric $(n=1,2,3,4 \ldots)$

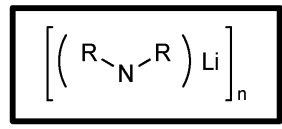

$\rightarrow$ broad selectivity ranges $\rightarrow$ temperature sensitive $\rightarrow$ energy intensive

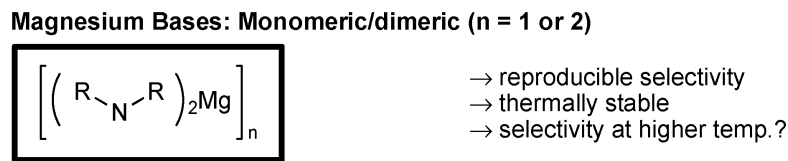

Fig. 1 Towards energy-effective asymmetric deprotonations: lithium vs. magnesium amides. 
describe an approach towards the development of both more generally efficient and, in addition, energy effective asymmetric deprotonations through the deployment of simple and readily accessible $C_{2}$-symmetric chiral magnesium bisamides.

Over recent years, we have probed the structure of magnesium bisamides in efforts to understand the key aspects that engender these complexes with comparatively enhanced levels of asymmetric efficiency (for example, complexes (R)-3-7, Scheme 1). ${ }^{10}$ While good levels of selectivity could be achieved in this benchmark asymmetric deprotonation process, based on some precedent within our laboratory, ${ }^{10 f}$ we envisaged that appropriate modification of the parent amine would lead to a highly utilisable process at less energy intensive temperatures, i.e. above $-78{ }^{\circ} \mathrm{C}$. From the outset, we considered two key items as essential for an energy-effective chiral base system: (i) the requisite parent amine must be simple in structure and, in turn, either readily prepared or, preferably, commercially available; and (ii) the system must operate at more readily used temperatures of $-40{ }^{\circ} \mathrm{C}$ or above.

Based on all of this, we next moved to evaluate the performance of the readily accessible $C_{2}$-symmetric magnesium amide $(R, R)-8$ (Scheme 1). Pleasingly, this complex displayed greatly improved enantioselectivity in the benchmark asymmetric deprotonation reaction, providing levels of enantioinduction greater than the corresponding lithium amide, including the typically more selective LiCl-based ate complex. ${ }^{13}$ Furthermore, the parent amine is structurally simple and is either readily prepared via the method of Alexakis et al. ${ }^{14}$ or can be purchased commercially.

Based on these initial screening results, base complex $(R, R)-\mathbf{8}$ was selected for further investigation. We next sought to ascertain the temperature dependence of our developing $C_{2}$-symmetric base system, in accordance with our design criteria. As such, we evaluated the performance of complex $(R, R)-\mathbf{8}$ at more elevated temperatures ranging from $-60{ }^{\circ} \mathrm{C}$ to $0{ }^{\circ} \mathrm{C}$ (Table 1). Remarkably, this $C_{2}$-symmetric amide maintained very good levels of enantioinduction over an almost $80{ }^{\circ} \mathrm{C}$ temperature range (entries $\left.1-5\right)$. In fact, base $(R, R)-8$
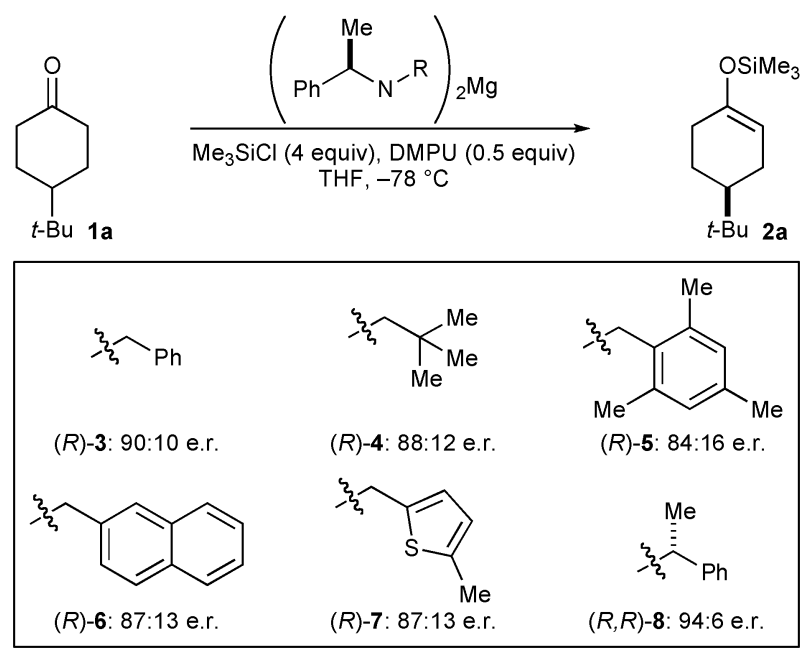

Scheme 1 Evaluation of simple Mg amides in the asymmetric deprotonation of 4-tert-butylcyclohexanone (1a). (Enantiomeric ratio given as $(S: R)$, DMPU $=$ 1,3-dimethyl-3,4,5,6-tetrahydro-2(1H)-pyrimidinone).
Table 1 Performance of base $(R, R)-\mathbf{8}$ over a range of temperatures in the asymmetric deprotonation of 4-tert-butylcyclohexanone (1a)

\begin{tabular}{llll}
\hline Entry & $T^{a}\left({ }^{\circ} \mathrm{C}\right)$ & Conv. $^{b}(\%)$ & e.r. $^{b}(S: R)$ \\
\hline 1 & -78 & 97 & $94: 6$ \\
2 & -60 & 93 & $92: 8$ \\
3 & -40 & 95 & $90: 10$ \\
4 & -20 & 97 & $88: 12$ \\
5 & 0 & 93 & $86: 14$ \\
6 & 23 & 89 & $75: 25$
\end{tabular}

${ }^{a}$ Bath temperature. ${ }^{b}$ Determined by G.C. analysis; see ESI.

demonstrates the same level of enantioselectivity at $0{ }^{\circ} \mathrm{C}$ as the analogous lithium complex achieves at $-78{ }^{\circ} \mathrm{C}$ (entry 5). $\ddagger$ It is also worth noting that this new reagent system is capable of delivering appreciable levels of enantioselection at room temperature $\left(23^{\circ} \mathrm{C}\right.$; entry 6$)$.

Encouraged by the degree of temperature tolerance of complex $(R, R)-\mathbf{8}$, we proceeded to further optimise the quantities of additive and silyl agent loading. Consequently, we found that only one equivalent of both DMPU and $\mathrm{Me}_{3}$ $\mathrm{SiCl}^{17,18}$ provided a chiral base system that could now deliver excellent asymmetric efficiency at $-78{ }^{\circ} \mathrm{C}$ for a range of substrates (Table 2, entries 1-5). Indeed, the levels of selectivity displayed here are amongst the highest levels obtained in such deprotonation reactions. More significantly, these experimental conditions have now also been shown to instil unprecedented levels of enantioinduction at $-20{ }^{\circ} \mathrm{C}$ (entries 6-10). These results are especially notable when considering the overall simplicity of the parent amine from which this reagent is derived. Additionally, yields of products were consistent regardless of the reaction temperature.

The unprecedented selectivity exhibited by this simple magnesium base system can perhaps be explained through the thermal stability and solution aggregation of magnesium amides in general. Magnesium amides typically exist in

Table 2 Performance of base $(R, R)-\mathbf{8}$ in the asymmetric deprotonation of prochiral ketones at -78 and $-20{ }^{\circ} \mathrm{C}$

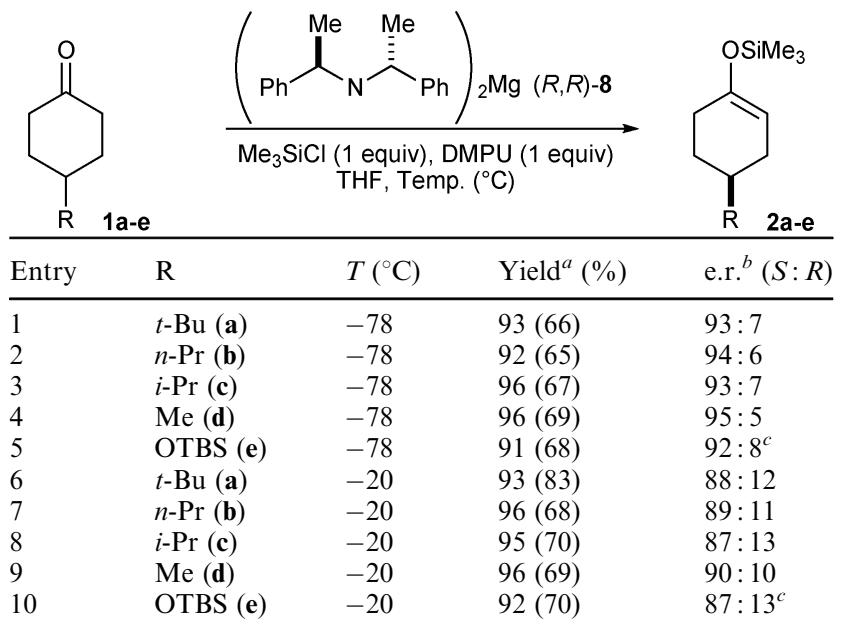

${ }^{a}$ Determined by G.C. analysis; values in parentheses are isolated yields of pure products. ${ }^{b}$ Determined by G.C. analysis. ${ }^{c}$ Determined by optical rotation; see ESI. 
solution as monomers or dimers. ${ }^{11}$ This is in stark contrast to lithium amides that are known to display a complex mixture of oligomeric forms that can have markedly different reactivity and selectivity profiles. ${ }^{12 b, c}$ Considering the small difference in selectivity of complex $(R, R)-\mathbf{8}$ across the temperature range applied, at present we postulate that the aggregation state of this magnesium bisamide does not differ drastically from -78 to $-20{ }^{\circ} \mathrm{C}$. As such, the structurally robust nature of this magnesium species serves to permit "warming" of the base solution without significant decomposition, and the observed temperature independence of the solution structure enables comparable selectivity at considerably more elevated temperatures. The exact nature of the active species is currently under investigation.

In summary, utilising unique properties characteristic of magnesium amides, we have successfully developed a novel $C_{2}$-symmetric magnesium bisamide and demonstrated its unique ability to function efficiently within asymmetric deprotonation reactions. Significantly, this new reagent operates extremely effectively at $-78{ }^{\circ} \mathrm{C}$, as well as the substantially more elevated $-20{ }^{\circ} \mathrm{C}$, maintaining very good levels of asymmetric efficiency and thereby presenting significant opportunities for energy conservation in this arena. Following these studies, we are currently focused on the development of further new chiral magnesium-centred bases to enable highly selective processes at ambient temperature.

We thank the EPSRC for funding (M.M.; EP/C548981/1) and the EPSRC Mass Spectrometry Service, University of Wales, Swansea, for analyses. We are also extremely grateful to Prof. Dr Klaus Ditrich (BASF SE, Ludwigshafen) for a generous supply of chiral amines.

\section{Notes and references}

$\ddagger$ The corresponding Li complex (lithium $(R, R)$-bis-(1-phenylethyl)amide) is reported to achieve an e.r. of $85: 15(S: R)$ at $-78{ }^{\circ} \mathrm{C} .{ }^{15}$ For comparison, we also evaluated this Li complex under conditions as established by Simpkins et al. ${ }^{13}$ using both internal quench (IQ) and external quench with $\mathrm{LiCl}$ additive ${ }^{16}$ (EQ) techniques, at $-20{ }^{\circ} \mathrm{C}$ and $0{ }^{\circ} \mathrm{C}$, with the following results (selectivity values given as $(S: R)$ ): $-20{ }^{\circ} \mathrm{C}: 77: 23$ (IQ), $79: 21$ (EQ); $0{ }^{\circ} \mathrm{C}: 76: 24$ (IQ), $74: 26$ (EQ).

1 Green chemistry guidelines include the consideration of the energetic cost of chemical processes, see: P. T. Anastas and J. C. Warner, Green Chemistry: Theory and Practice, Oxford University Press, New York, 2000.

2 For the energetic consideration of selected chemical reactions, see: M. J. Gronnow, R. J. White, J. H. Clark and D. J. Macquarrie, Org. Process Res. Dev., 2005, 9, 516.

3 D. Hayes, Synthetic Chemistry, Pre-Clinical Development, Chemical Development, GlaxoSmithKline, personal communication.

4 For examples, see: (a) P. Knochel, W. Dohle, N. Gommermann, F. F. Kneisel, F. Kopp, T. Korn, I. Sapountzis and V. A. Vu, Angew. Chem., Int. Ed., 2003, 42, 4302; (b) H. Ila, O. Baron, A. J. Wagner and P. Knochel, Chem. Commun., 2006, 583; (c) T. D. Blümke, F. M. Piller and P. Knochel, Chem. Commun., 2010, 46, 4082; (d) S. H. Wunderlich, C. J. Rohbogner, A. Unsinn and P. Knochel, Org. Process Res. Dev., 2010, 14, 339.

5 For selected examples, see: (a) M. R. Netherton, C. Dai, K. Neuschütz and G. C. Fu, J. Am. Chem. Soc., 2001, 123, 10099; (b) A. Leitner, S. Shekhar, M. J. Pouy and J. F. Hartwig, J. Am. Chem. Soc., 2005, 127, 15506; (c) T. Hama, D. A. Culkin and J. F. Hartwig, J. Am. Chem. Soc., 2006, 128, 4976; (d) O. Navarro, N. Marion, J. Mei and S. P. Nolan, Chem.-Eur. J., 2006, 12, 5142; (e) L. Melzig, A. Metzger and P. Knochel,
J. Org. Chem., 2010, 75, 2131; (f) S. Çalimsiz, M. Sayah, D. Mallik and M. G. Organ, Angew. Chem., Int. Ed., 2010, 49, 2014.

6 For a review, see: N. S. Simpkins, Top. Stereochem., 2010, 26, 1.

7 For the use of enol silanes in organic synthesis, see: (a) P. Brownbridge, Synthesis, 1983, 1; (b) P. Brownbridge, Synthesis, 1983, 85; (c) S. Kobayashi, K. Manabe, H. Ishitani and J.-I. Matsuo, Silyl Enol Ethers in Science of Synthesis, ed. I. Fleming, Thieme, Stuttgart, 2001, vol. 4, p. 317.

8 For examples of effective chiral lithium amide bases, see: (a) R. Shirai, M. Tanaka and K. Koga, J. Am. Chem. Soc., 1986, 108, 543; (b) R. P. C. Cousins and N. S. Simpkins, Tetrahedron Lett., 1989, 30, 7241; (c) K. Aoki, H. Noguchi, K. Tomioka and K. Koga, Tetrahedron Lett., 1993, 34, 5105; (d) B. J. Bunn and N. S. Simpkins, J. Org. Chem., 1993, 58, 533; (e) B. J. Bunn, P. J. Cox and N. S. Simpkins, Tetrahedron, 1993, 49, 207; $(f)$ R. Shirai, D. Sato, K. Aoki, M. Tanaka, H. Kawasaki and K. Koga, Tetrahedron, 1997, 53, 5963; $(g)$ K. Aoki and K. Koga, Tetrahedron Lett., 1997, 38, 2505; (h) V. K. Aggarwal, P. S. Humphries and A. Kenwick, J. Chem. Soc., Perkin Trans. 1, 1999, 2883; (i) C.-D. Graf, C. Malan and P. Knochel, Angew. Chem., Int. Ed., 1998, 37, 3014; (j) C.-D. Graf, C. Malan, K. Harms and P. Knochel, J. Org. Chem., 1999, 64, 5581; (k) K. Aoki and K. Koga, Chem. Pharm. Bull., 2000, 48, 571; (l) J. Busch-Petersen and E. J. Corey, Tetrahedron Lett., 2000, 41, 6941.

9 For examples, see: D. M. Hodgson, A. R. Gibbs and G. P. Lee, Tetrahedron, 1996, 52, 14361. See also ref. 6.

10 (a) K. W. Henderson, W. J. Kerr and J. H. Moir, Chem. Commun., 2000, 479; (b) K. W. Henderson, W. J. Kerr and J. H. Moir, Synlett, 2001, 1253; (c) K. W. Henderson, W. J. Kerr and J. H. Moir, Chem. Commun., 2001, 1722; (d) J. D. Anderson, P. García García, D. Hayes, K. W. Henderson, W. J. Kerr, J. H. Moir and K. P. Fondekar, Tetrahedron Lett., 2001, 42, 7111; (e) K. W. Henderson, W. J. Kerr and J. H. Moir, Tetrahedron, 2002, 58, 4573; $(f)$ E. L. Carswell, D. Hayes, K. W. Henderson, W. J. Kerr and C. J. Russell, Synlett, 2003, 1017; $(g)$ M. J. Bassindale, J. J. Crawford, K. W. Henderson and W. J. Kerr, Tetrahedron Lett., 2004, 45, 4175.

11 (a) P. E. Eaton, H. Higuchi and R. Millikan, Tetrahedron Lett., 1987, 28, 1055; (b) P. E. Eaton, C. H. Lee and Y. Xiong, J. Am. Chem. Soc., 1989, 111, 8016; (c) J. F. Allan, K. W. Henderson and A. R. Kennedy, Chem. Commun., 1999, 1325; (d) M. Westerhausen, Inorg. Chem., 1991, 30, 96; (e) W. Clegg, F. J. Craig, K. W. Henderson, A. R. Kennedy, R. E. Mulvey, P. A. O'Neil and D. Reed, Inorg. Chem., 1997, 36, 6238; (f) K. W. Henderson and W. J. Kerr, Chem.-Eur. J., 2001, 7, 3430; $(g)$ B. Conway, E. Hevia, A. R. Kennedy, R. E. Mulvey and S. Weatherstone, Dalton Trans., 2005, 1532.

12 For a review on the structure of Li amides, see: $(a)$ R. E. Mulvey, Chem. Soc. Rev., 1991, 20, 167. For the impact of Li amide oligomers on the selectivity of asymmetric deprotonation processes, see: (b) D. Sato, H. Kawasaki, I. Shimada, Y. Arata, K. Okamura, T. Date and K. Koga, J. Am. Chem. Soc., 1992, 114, 761; (c) K. Aoki, K. Tomioka, H. Noguchi and K. Koga, Tetrahedron, 1997, 53, 13641.

13 The highest level of enantioselectivity recorded for the corresponding Li base with $\mathrm{LiCl}$ at $-78{ }^{\circ} \mathrm{C}$ is $91.5: 8.5(S: R)$. See: B. J. Bunn, N. S. Simpkins, Z. Spavold and M. J. Crimmin, J. Chem. Soc., Perkin Trans. 1, 1993, 3113 and ref. $8 d$.

14 A. Alexakis, S. Gille, F. Prian, S. Rosset and K. Ditrich, Tetrahedron Lett., 2004, 45, 1449.

15 N. S. Simpkins, J. Chem. Soc., Chem. Commun., 1986, 88.

16 The use of an $\mathrm{LiCl}$ additive is known to enable the highest levels of selectivity in externally quenched reactions. ${ }^{6,13}$

17 For the use of only one equivalent of $\mathrm{Me}_{3} \mathrm{LiCl}$ as an internal quench within deprotonation processes, see: (a) W. J. Kerr, A. J. B. Watson and D. Hayes, Chem. Commun., 2007, 5049; (b) W. J. Kerr, A. J. B. Watson and D. Hayes, Org. Biomol. Chem., 2008, 6, 1238; (c) W. J. Kerr, A. J. B. Watson and D. Hayes, Synlett, 2008, 1386.

18 Such internal quench protocols usually require an excess of electrophile, see: E. J. Corey and A. W. Gross, Tetrahedron Lett., 1984, 25, 495. 